\title{
COMMUNICATION
}

\section{La chéilite granulomateuse de Miescher : A propos d'un cas}

\author{
Lan $\mathbf{R}^{1}$, Garçonnet $\mathrm{J}^{1}$, Massereau $\mathrm{E}^{1}$, Catherine $\mathrm{JH}^{1,2}$ \\ 1 - Service de chirurgie orale, CHU Timone, Marseille \\ 2 - UMR 7268 ADES Aix-Marseille Université/EFS/CNRS, Marseille
}

Mots Clés : chéilite, granulomatose de Miescher, granulomatose oro-faciale

La chéilite granulomateuse de Miescher (CGM), décrite pour la première fois par Miescher en 1945, est une pathologie inflammatoire rare dont l'étiologie n'est pas encore connue et se manifestant par une tuméfaction labiale d'une ou des deux lèvres provoquant une macrochéilite. Elle peut être retrouvée en présence d'une paralysie faciale, d'une langue plicaturée et d'un œdème oro-facial pouvant alors évoquer le syndrome de Melkerson-Rosenthal (SMR); ces deux pathologies pouvant aussi être considérées comme des granulomatoses oro-faciales.

Une patiente de 23 ans a été adressée pour une tuméfaction chronique de la lèvre supérieure apparue depuis plusieurs mois avec douleurs permanentes et irradiantes. L'examen clinique révélait une tuméfaction généralisée de la lèvre supérieure avec légère desquamation. Aucune autre lésion muqueuse ou paralysie faciale n'ont été objectivées. Un traitement de prednisolone et metronidazole pendant 1 mois a été instauré sans succès par son médecin généraliste. Une biopsie a été réalisée et l'examen histologique a révélé la présence de granulomes gigantocellulaires épithélioïdes sans nécrose caséeuse. Les examens biologiques, paracliniques et le bilan étiologique n'ont pas permis de retenir assez d'éléments pour évoquer une maladie de Crohn, un œdème allergique ou d'autres maladies systémiques comme la sarcoïdose ou le lupus. Des éléments d'auto-immunité biologique avec des anticorps antinucléaires au seuil de positivité avec spécificité anti SM et anti RNP ont toutefois été retrouvés. Le diagnostic provisoire de chéilite granulomatose de Miescher a été retenu. En raison de ce terrain immunologique, un traitement par hydroxychloroquine (Plaquenil囚 $200 \mathrm{mg}$ ) a été instauré permettant au bout de 3 mois une légère diminution de la tuméfaction labiale non satisfaisante pour la patiente. II était alors décidé de pratiquer des injections de triamcinolone acétonide $40 \mathrm{mg}$ retard (Kenacort ${ }^{\circledR}$ ) toutes les 3 semaines dans le but d'obtenir une rémission totale de la lésion. En l'absence de nouveaux signes en faveur d'un SMR ou d'une maladie de Crohn, le diagnostic définitif de CGM a été retenu.

La difficulté diagnostique réside dans le fait que la CGM peut être une manifestation buccale d'une maladie systémique telle que la sarcoïdose, la maladie de Crohn, plus rarement la maladie de Wegener ou encore la tuberculose. La lésion histopathologique est caractérisée par la présence de granulomes giganto-cellulaires épithélioïdes sans nécrose caséeuse. Du fait de la méconnaissance de l'étiologie, de nombreux traitements ont été proposés tels que la clofazine, les anticorps monoclonaux, les corticoïdes per-os ou encore les injections intra-lésionnelles 
de corticoïdes qui se sont révélées efficaces dans notre cas clinique. Une chirurgie plastique esthétique pourra être proposée en cas d'échecs ou de séquelles de traitements médicamenteux.

\section{Références}

Bacci C et al. J Eur Acad Dermatol Venereol 2010;24:363-4.

Banks T et al. Br J Dermatol 2012;166:934-7.

Sciubba JJ et al. J Oral Pathol Med 2003;32:576-85. 